\title{
49. HIGH-RESOLUTION MAGNETIC SUSCEPTIBILITY AND CARBONATE RECORDS: SOUTHERN KERGUELEN PLATEAU ${ }^{1}$
}

\author{
Wolfgang U. Dorn ${ }^{2}$
}

\begin{abstract}
High-resolution (5-cm interval) whole-core magnetic susceptibility measurements were made during ODP Leg 119 for Sites 744 and 745, located on the Southern Kerguelen Plateau. The magnetic log of Hole 744B is completed by carbonate analyses based on tightly spaced samples ( 7 samples per section; approximately 20 -cm intervals). Initial results indicate an inverse relationship between susceptibility values and percent carbonate. The data sets represent a potential base for within-site hole cross-correlations and global paleoenvironmental studies.
\end{abstract}

\section{INTRODUCTION}

The logging of whole-core magnetic susceptibility (WCMS) has lately become an increasingly routine part among ODP shipboard measurements. Partially this is due to the fact that the shipboard susceptibility meter is user-friendly and allows simple, rapid, and nondestructive measurements of complete sections at relatively narrow intervals. Magnetic susceptibility is a function of the concentration and composition of magnetic components within the sediment, and thus WCMS logs reflect lithological variations (with possible exceptions in the presence of bacterial magnetite or drilling contaminations). The usefulness of magnetic susceptibility measurements as proxy indicators for paleoenvironmental changes has been well established (e.g., Thompson et al., 1980; Robinson, 1986, in press; Bloemendal et al., 1988a).

\section{INSTRUMENTATION AND METHODS}

WCMS measurements were applied on split core sections recovered with the advanced piston corer (APC) from Holes 744A, 744B, 744C, and 745B aboard JOIDES Resolution. The APC-drilled sections were measured using the Bartington Instruments M.S.1 susceptibility meter at high-sensitivity $(0.1)$ and low-frequency $(0.47 \mathrm{kHz})$ settings (for details see Bloemendal et al., 1988b; Robinson, in press). The split core sections were pushed manually through the Bartington Instruments 80$\mathrm{mm}$ inside-diameter loop-type susceptibility sensor and measurements were performed successively downcore, usually at 5 $\mathrm{cm}$ intervals. Section boundaries (tops and bottoms), as well as obviously disturbed section intervals, were not measured. The measurements were stored on floppy disks on the interfaced work-station microcomputer.

Shore-based laboratory carbonate analyses were performed on tightly spaced (7 samples per section) shipboard samples of 5 $\mathrm{cm}^{3}$ volume from Hole 744B. Calcium carbonate values were determined by applying both coulometric and spectrometric techniques. The freeze-dried samples were pulverized with a swing-grinding mill and measured on either a Ströhlein COULOMAT $701 \mathrm{LI}$ or LECO CS 244 carbonate analyzer. To

\footnotetext{
${ }^{1}$ Barron, J., Larsen, B., et al., 1991. Proc. ODP, Sci. Results, 119: College Station, TX (Ocean Drilling Program).

${ }^{2}$ Hawaii Institute of Geophysics, University of Hawaii, Honolulu, HI 96822 U.S.A., and Geologisch-Paläontologisches Institut, Universität Kiel, D-2300 Kiel, Federal Republic of Germany (present address: Geologisch-Paläontologisches Institut und Museum, Universität Kiel, Olshausenstrasse 40, D-2300 Kiel, Federa Republic of Germany).
}

enhance accuracy, most values represent arithmetic means of double or triple individual measurements. For the presentation of carbonate percentages vs. depth plots, original sample-depth assignments have been recalculated, taking into account drill recovery exceeding $100 \%$.

\section{RESULTS}

Magnetic susceptibility logs of Site 744 are shown in Figure 1A through $1 \mathrm{C}$ divided into 20 -m intervals for each hole. Relatively higher volume magnetic susceptibility values in the uppermost sections, between about 23 and $12 \mathrm{~m}$ below seafloor (mbsf) apparently correspond to a major lithological change within each hole; at 21.60, 21.25, and $23.00 \mathrm{mbsf}$ (Barron, Larsen, et al., 1989) in Holes 744A, 744B, and 744C, respectively, a shift occurs from mainly diatom ooze to calcareous nannofossil ooze sediments. In the deeper calcareous sedimentary unit, a comparison between WCMS log and high-resolution calcium carbonate analyses for Hole 744B (Fig. 2) reveals an inverse relationship between carbonate percentages and magnetic susceptibility values. Such a relationship is attributed to fluctuations in the ratio of biogenic to lithogenic components: if the amount of magnetic material within the sediment is assumed to remain constant while the supply of biogenic carbonate varies, then, as a consequence of dilution, higher carbonate percentages should result in lowered susceptibility values, and vice versa. Therefore, susceptibility curve variations in nannofossil sediments of Hole 744B can reflect changes in the supply of calcium carbonate, as well as increased lithogenic input or possible carbonate dissolution. Individual results of the carbonate analyses are listed in Table 1.

The WCMS profiles for Hole 745B (Fig. 3) show a pronounced cyclicity on a scale of 1-2 m, especially distinct in the interval from about 50 to $100 \mathrm{mbsf}$. The sequence recovered at Site 745 consists almost entirely of diatom ooze and diatomaceous clay, with practically no carbonate components. The diatomaceous sediments are characterized by alternations of variably clay-rich zones. Variations of WCMS values are thus tentatively ascribed to fluctuations in the ratios of biogenic siliceous components and clay content.

\section{ACKNOWLEDGMENTS}

I am grateful to J. Barron and J. Baldauf for encouraging susceptibility and carbonate measurements. J. Tauxe provided valuable technical assistance and interest during the shipboard analyses. S. Mühlhan and C. Hensen helped perform the carbonate analyses. 
Volume magnetic susceptibility $\left(10^{-6} \mathrm{cgs}\right)$
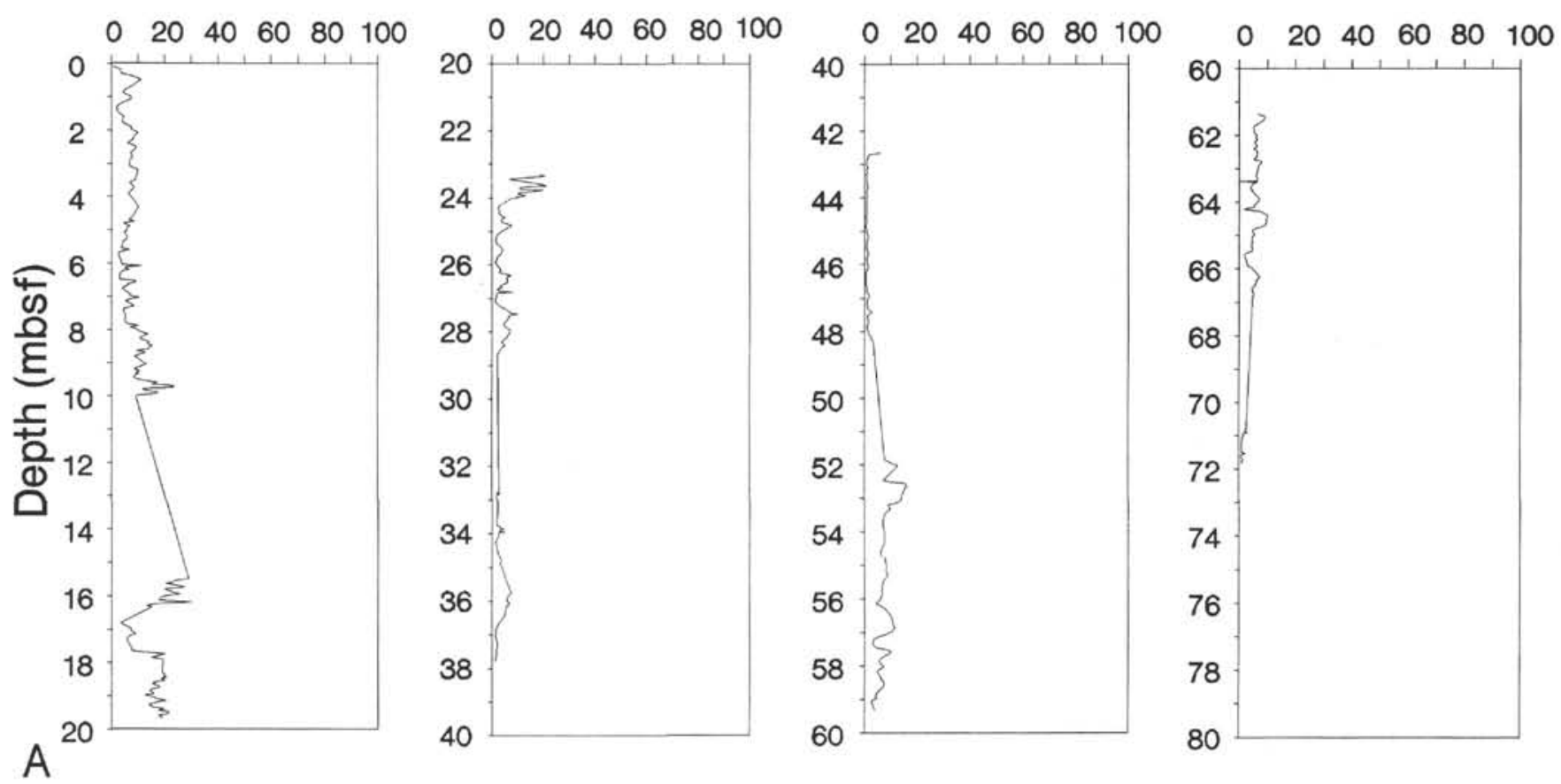

\section{Volume magnetic susceptibility $\left(10^{-6} \mathrm{cgs}\right)$}
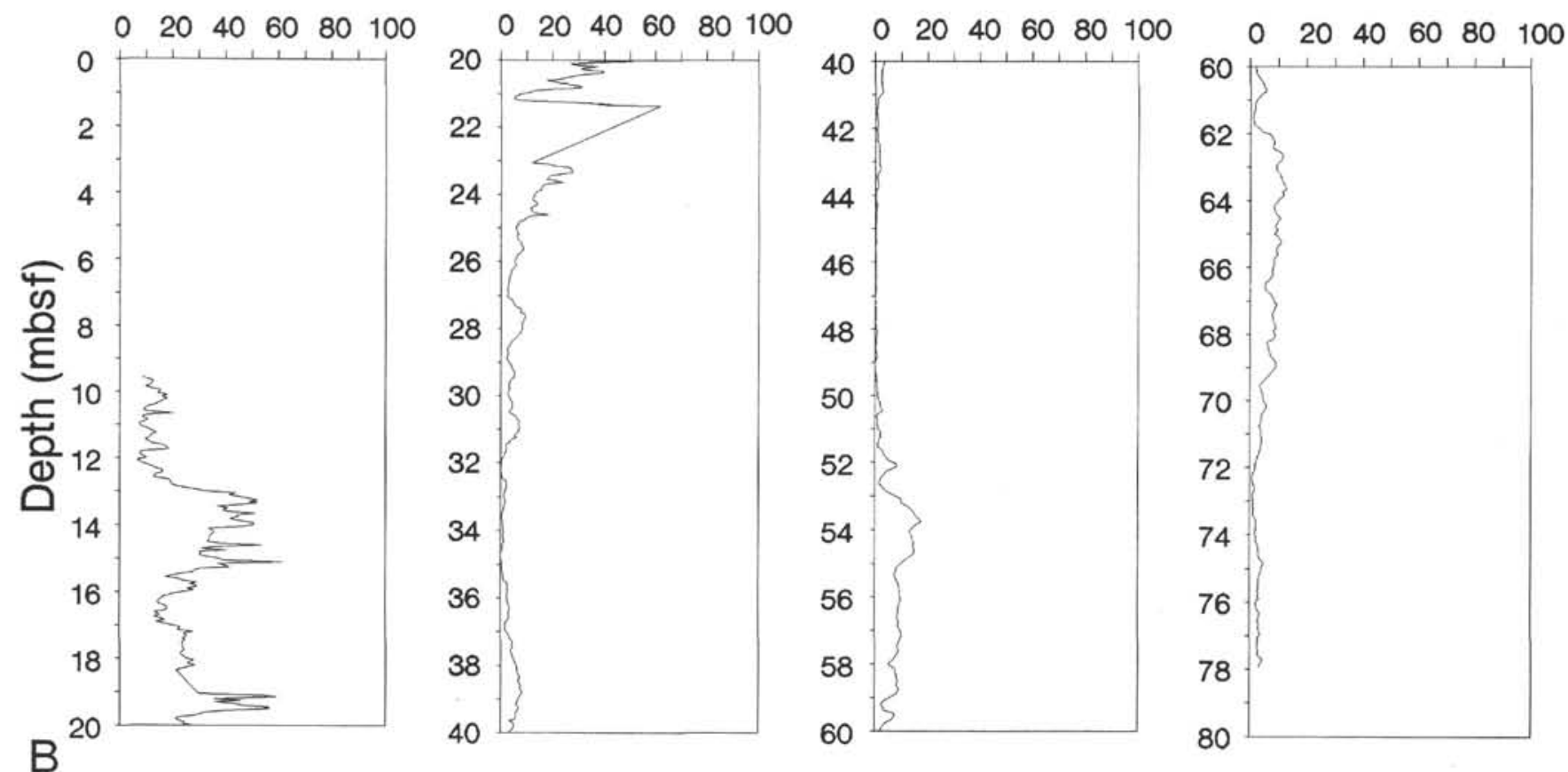

Figure 1. Volume magnetic susceptibility logs of Holes 744A (A), 744B (B), and 744C (C). Logs are divided into 20-m intervals. 


\section{Volume magnetic susceptibility $\left(10^{-6} \mathrm{cgs}\right)$}
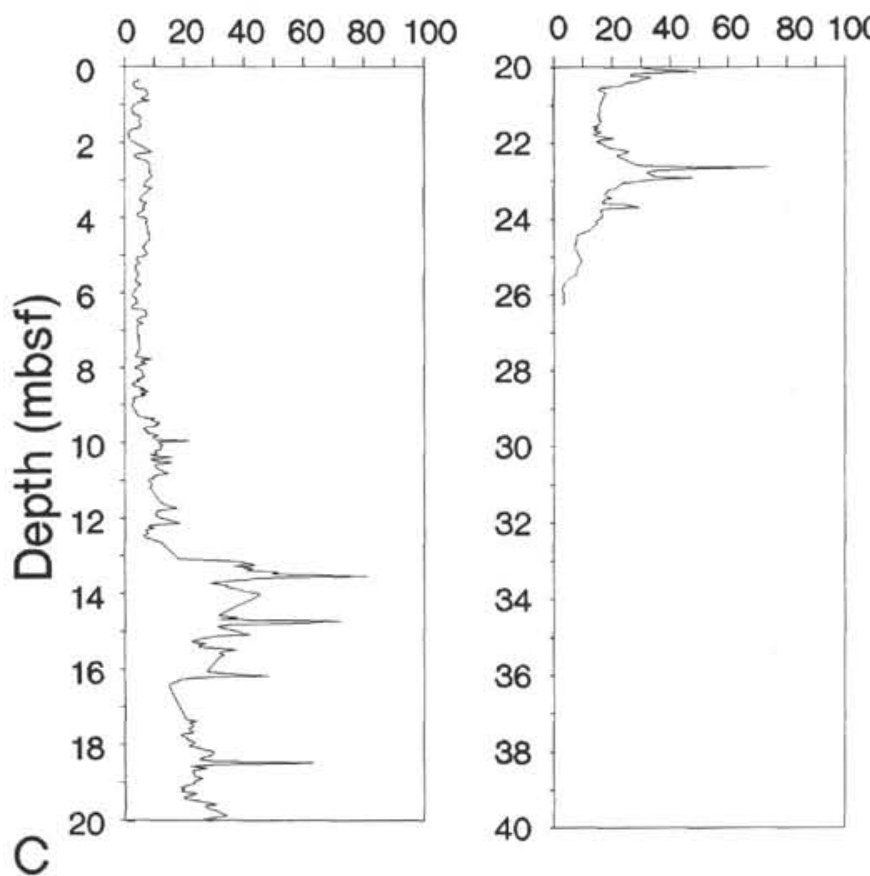

Figure 1 (continued).

\section{REFERENCES}

Barron, J., Larsen, B., et al., 1989. Proc. ODP, Init. Repts., 119: College Station, TX (Ocean Drilling Program).

Bloemendal, J., Lamb, B., and King, J., 1988a. Paleoenvironmental implications of rock-magnetic properties of late Quaternary sediment cores from the eastern Equatorial Atlantic. Paleoceanography, 3:6187.

Bloemendal, J., Tauxe, L., Valet, J.-P., and Shipboard Scientific Party, 1988b. High-resolution, whole-core magnetic susceptibility logs from Leg 108. In Ruddiman, W., Sarnthein, M., et al., Proc. ODP, Init. Repts., 108: College Station, TX (Ocean Drilling Program), 10051013.

Robinson, S. G., 1986. The late Pleistocene palaeoclimatic record of North Atlantic deep-sea sediments revealed by mineral-magnetic measurements. Phys. Earth Planet. Inter., 42:22-47.

, in press. Applications for whole-core magnetic susceptibility measurements of deep-sea sediments: Ocean Drilling Program Leg 115 results. In Backman, J., Duncan, R. A., et al., Proc. ODP, Sci. Results, 115: College Station, TX (Ocean Drilling Program).

Thompson, R., Bloemendal, J., Dearing, J. A., Oldfield, F., Rummery, T. A., Stober, J. C., and Turner, G. M., 1980. Environmental applications of magnetic measurements. Science, 207:481-486

Date of initial receipt: 27 November 1989

Date of acceptance: 4 June 1990

Ms 119B-199 


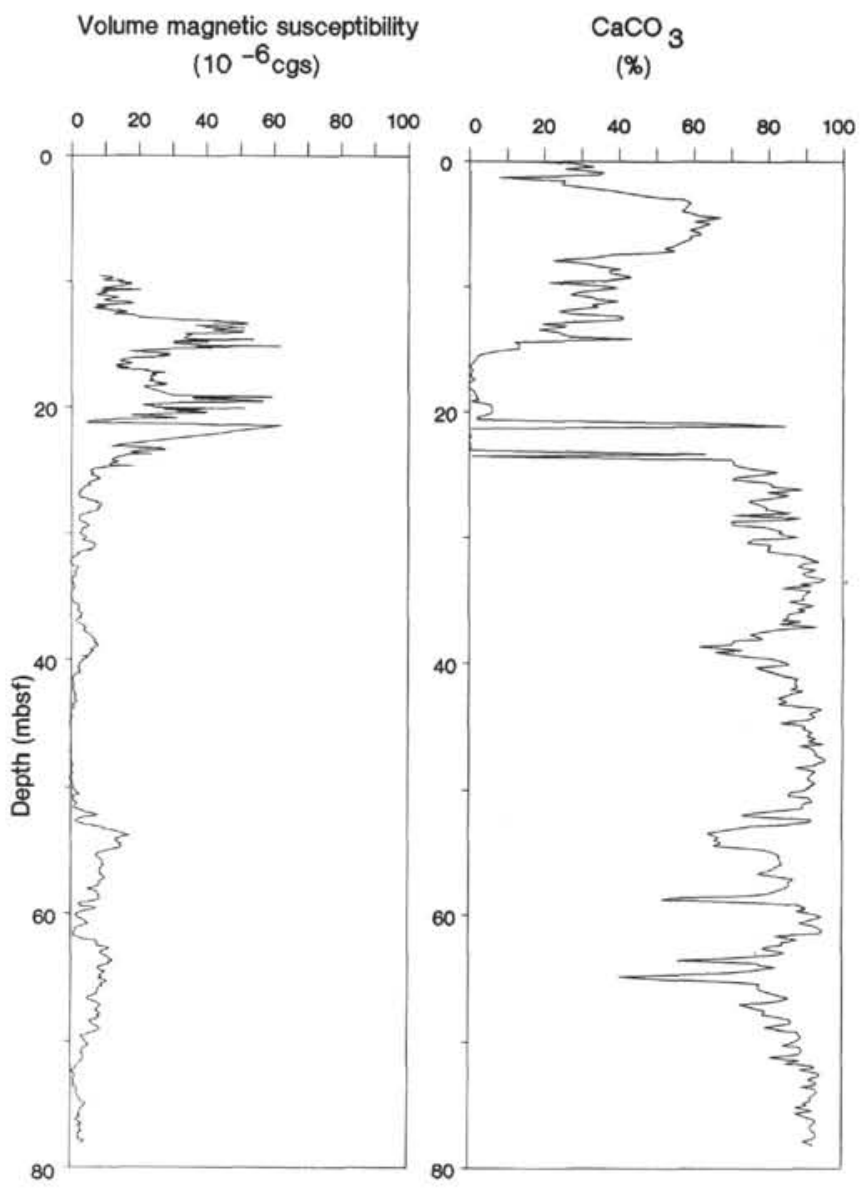

Figure 2. Volume magnetic susceptibility $\log$ and percent calcium carbonate curve of Hole 744B. Carbonate curve based on analyses listed in Table 1. Comparison reveals an inverse relationship between carbonate content and susceptibility values.
Table 1. Calcium carbonate percentages of all samples analyzed from Hole 744B.

\begin{tabular}{|c|c|c|}
\hline $\begin{array}{l}\text { Core, section, } \\
\text { interval }(\mathrm{cm})\end{array}$ & $\begin{array}{l}\text { Depth } \\
\text { (mbsf) }\end{array}$ & $\begin{array}{c}\mathrm{CaCO}_{3} \\
(\%)\end{array}$ \\
\hline $1 \mathrm{H}-1,0-1$ & 0.00 & 16.3 \\
\hline $1 \mathrm{H}-1,16-17$ & 0.16 & 27.3 \\
\hline $1 \mathrm{H}-1,46-47$ & 0.46 & 33.1 \\
\hline $1 \mathrm{H}-1,63-64$ & 0.63 & 25.7 \\
\hline $1 \mathrm{H}-1,94-95$ & 0.94 & 35.9 \\
\hline $1 \mathrm{H}-1,114-115$ & 1.14 & 34.2 \\
\hline $1 \mathrm{H}-1,136-137$ & 1.36 & 7.7 \\
\hline $1 \mathrm{H}-2,0-1$ & 1.50 & 14.9 \\
\hline $1 \mathrm{H}-2,16-17$ & 1.66 & 25.5 \\
\hline $1 \mathrm{H}-2,46-47$ & 1.96 & 24.7 \\
\hline $1 \mathrm{H}-2,63-64$ & 2.13 & 28.6 \\
\hline $1 \mathrm{H}-2,94-95$ & 2.44 & 38.2 \\
\hline $1 \mathrm{H}-2,114-115$ & 2.64 & 41.9 \\
\hline $1 \mathrm{H}-2,136-137$ & 2.86 & 47.1 \\
\hline $1 \mathrm{H}-3,0-1$ & 3.00 & 50.5 \\
\hline $1 \mathrm{H}-3,16-17$ & 3.16 & 57.1 \\
\hline $1 \mathrm{H}-3,46-47$ & 3.46 & 59.0 \\
\hline $1 \mathrm{H}-3,63-64$ & 3.63 & 58.6 \\
\hline $1 \mathrm{H}-3,94-95$ & 3.94 & 57.5 \\
\hline $1 \mathrm{H}-3,114-115$ & 4.14 & 56.7 \\
\hline $1 \mathrm{H}-3,136-137$ & 4.36 & 60.5 \\
\hline $1 \mathrm{H}-4,0-1$ & 4.50 & 61.0 \\
\hline $1 \mathrm{H}-4,16-17$ & 4.66 & 67.1 \\
\hline $1 \mathrm{H}-4,46-47$ & 4.96 & 60.1 \\
\hline $1 \mathrm{H}-4,63-64$ & 5.13 & 64.2 \\
\hline $1 \mathrm{H}-4,94-95$ & 5.44 & 61.6 \\
\hline $1 \mathrm{H}-4,114-115$ & 5.64 & 58.8 \\
\hline $1 \mathrm{H}-4,136-137$ & 5.86 & 61.5 \\
\hline $1 \mathrm{H}-5,0-1$ & 6.00 & 61.9 \\
\hline $1 \mathrm{H}-5,16-17$ & 6.16 & 59.1 \\
\hline $1 \mathrm{H}-5,46-47$ & 6.46 & 59.1 \\
\hline $1 \mathrm{H}-5,63-64$ & 6.63 & 57.2 \\
\hline $1 \mathrm{H}-5,94-95$ & 6.94 & 55.2 \\
\hline $1 \mathrm{H}-5,114-115$ & 7.14 & 52.1 \\
\hline $1 \mathrm{H}-5,136-137$ & 7.36 & 54.8 \\
\hline $1 \mathrm{H}-6,0-1$ & 7.50 & 50.1 \\
\hline $1 \mathrm{H}-6,16-17$ & 7.66 & 38.8 \\
\hline $1 \mathrm{H}-6,46-47$ & 7.96 & 32.8 \\
\hline $1 \mathrm{H}-6,63-64$ & 8.13 & 22.3 \\
\hline 1H-6, 94-95 & 8.44 & 31.9 \\
\hline $1 \mathrm{H}-6,114-115$ & 8.64 & 34.5 \\
\hline $1 \mathrm{H}-6,136-137$ & 8.86 & 40.3 \\
\hline $1 \mathrm{H}-7,0-1$ & 9.00 & 37.6 \\
\hline $1 \mathrm{H}-7,16-17$ & 9.16 & 37.5 \\
\hline $1 \mathrm{H}-7,46-47$ & 9.46 & 43.0 \\
\hline $2 \mathrm{H}-1,0-1$ & 9.50 & 35.6 \\
\hline $2 \mathrm{H}-1,16-17$ & 9.66 & 21.2 \\
\hline $2 \mathrm{H}-1,46-47$ & 9.96 & 36.3 \\
\hline $2 \mathrm{H}-1,63-64$ & 10.13 & 39.5 \\
\hline $2 \mathrm{H}-1,94-95$ & 10.44 & 29.4 \\
\hline $2 \mathrm{H}-1,114-115$ & 10.64 & 27.0 \\
\hline $2 \mathrm{H}-1,136-137$ & 10.86 & 33.1 \\
\hline $2 \mathrm{H}-2,0-1$ & 11.00 & 33.7 \\
\hline $2 \mathrm{H}-2,16-17$ & 11.16 & 39.7 \\
\hline $2 \mathrm{H}-2,46-47$ & 11.46 & 32.8 \\
\hline $2 \mathrm{H}-2,63-64$ & 11.63 & 34.3 \\
\hline $2 \mathrm{H}-2,94-95$ & 11.94 & 24.0 \\
\hline $2 \mathrm{H}-2,114-115$ & 12.14 & 27.0 \\
\hline $2 \mathrm{H}-2,136-137$ & 12.36 & 39.9 \\
\hline $2 \mathrm{H}-3,0-1$ & 12.50 & 41.3 \\
\hline $2 \mathrm{H}-3,16-17$ & 12.66 & 40.2 \\
\hline $2 \mathrm{H}-3,46-47$ & 12.96 & 19.5 \\
\hline $2 \mathrm{H}-3,63-64$ & 13.13 & 26.0 \\
\hline $2 \mathrm{H}-3,94-95$ & 13.44 & 18.4 \\
\hline $2 \mathrm{H}-3,114-115$ & 13.64 & 23.9 \\
\hline $2 \mathrm{H}-3,136-137$ & 13.86 & 25.1 \\
\hline $2 \mathrm{H}-4,0-1$ & 14.00 & 26.8 \\
\hline $2 \mathrm{H}-4,16-17$ & 14.16 & 43.3 \\
\hline $2 \mathrm{H}-4,46-47$ & 14.46 & 12.1 \\
\hline $2 \mathrm{H}-4,63-64$ & 14.63 & 13.2 \\
\hline $2 \mathrm{H}-4,94-95$ & 14.94 & 13.3 \\
\hline $2 \mathrm{H}-4,114-115$ & 15.14 & 7.8 \\
\hline $2 \mathrm{H}-4,136-137$ & 15.36 & 4.5 \\
\hline $2 \mathrm{H}-5,0-1$ & 15.50 & 2.3 \\
\hline $2 \mathrm{H}-5,16-17$ & 15.66 & 2.2 \\
\hline $2 \mathrm{H}-5,46-47$ & 15.96 & 1.4 \\
\hline $2 \mathrm{H}-5,63-64$ & 16.13 & 0.8 \\
\hline
\end{tabular}

\begin{tabular}{|c|c|c|}
\hline $\begin{array}{l}\text { Core, section, } \\
\text { interval }(\mathrm{cm})\end{array}$ & $\begin{array}{l}\text { Depth } \\
\text { (mbsf) }\end{array}$ & $\begin{array}{c}\mathrm{CaCO}_{3} \\
(\%)\end{array}$ \\
\hline $2 \mathrm{H}-5,94-95$ & 16.44 & 0.0 \\
\hline $2 \mathrm{H}-5,114-115$ & 16.64 & 0.9 \\
\hline $2 \mathrm{H}-5,136-137$ & 16.86 & 0.5 \\
\hline $2 \mathrm{H}-6,0-1$ & 17.00 & 0.4 \\
\hline $2 \mathrm{H}-6,16-17$ & 17.16 & 0.2 \\
\hline $2 \mathrm{H}-6,46-47$ & 17.46 & 1.5 \\
\hline $2 \mathrm{H}-6,63-64$ & 17.63 & 0.2 \\
\hline $2 \mathrm{H}-6,94-95$ & 17.94 & 0.0 \\
\hline $2 \mathrm{H}-6,114-115$ & 18.14 & 0.1 \\
\hline $2 \mathrm{H}-6,136-137$ & 18.36 & 1.0 \\
\hline $3 \mathrm{H}-1,0-1$ & 19.00 & 2.3 \\
\hline $3 \mathrm{H}-1,16-17$ & 19.16 & 0.4 \\
\hline $3 \mathrm{H}-1,46-47$ & 19.46 & 5.7 \\
\hline $3 \mathrm{H}-1,63-64$ & 19.63 & 5.9 \\
\hline $3 \mathrm{H}-1,94-95$ & 19.94 & 6.1 \\
\hline $3 \mathrm{H}-1,114-115$ & 20.14 & 6.0 \\
\hline $3 \mathrm{H}-1,136-137$ & 20.36 & 4.2 \\
\hline $3 \mathrm{H}-2,0-1$ & 20.50 & 1.9 \\
\hline $3 \mathrm{H}-2,16-17$ & 20.66 & 2.1 \\
\hline $3 \mathrm{H}-2,46-47$ & 20.96 & 67.9 \\
\hline $3 \mathrm{H}-2,63-64$ & 21.13 & 84.6 \\
\hline $3 \mathrm{H}-\mathrm{CC}, 1-2$ & 21.39 & 0.3 \\
\hline $4 \mathrm{H}-1,0-1$ & 21.50 & 0.0 \\
\hline $4 \mathrm{H}-1,46-47$ & 21.96 & 0.3 \\
\hline $4 \mathrm{H}-1,63-64$ & 22.13 & 0.1 \\
\hline $4 \mathrm{H}-1,94-95$ & 22.44 & 0.0 \\
\hline $4 \mathrm{H}-1,114-115$ & 22.64 & 0.2 \\
\hline $4 \mathrm{H}-1,136-137$ & 22.86 & 0.2 \\
\hline $4 \mathrm{H}-2,0-1$ & 23.00 & 0.3 \\
\hline $4 \mathrm{H}-2,16-17$ & 23.16 & 0.0 \\
\hline $4 \mathrm{H}-2,46-47$ & 23.46 & 63.4 \\
\hline $4 \mathrm{H}-2,63-64$ & 23.63 & 0.5 \\
\hline $4 \mathrm{H}-2,94-95$ & 23.94 & 70.2 \\
\hline $4 \mathrm{H}-2,114-115$ & 24.14 & 70.9 \\
\hline $4 \mathrm{H}-2,136-137$ & 24.36 & 70.8 \\
\hline $4 \mathrm{H}-3,0-1$ & 24.50 & 72.9 \\
\hline $4 \mathrm{H}-3,16-17$ & 24.66 & 75.1 \\
\hline $4 \mathrm{H}-3,46-47$ & 24.94 & 82.6 \\
\hline $4 \mathrm{H}-3,63-64$ & 25.13 & 79.0 \\
\hline $4 \mathrm{H}-3,94-95$ & 25.44 & 71.5 \\
\hline $4 \mathrm{H}-3,114-115$ & 25.64 & 70.5 \\
\hline $4 \mathrm{H}-3,136-137$ & 25.86 & 79.9 \\
\hline $4 \mathrm{H}-4,0-1$ & 26.00 & 81.1 \\
\hline $4 \mathrm{H}-4,16-17$ & 26.16 & 81.0 \\
\hline $4 \mathrm{H}-4,46-47$ & 26.46 & 89.2 \\
\hline $4 \mathrm{H}-4,63-64$ & 26.63 & 80.0 \\
\hline $4 \mathrm{H}-4,94-95$ & 26.94 & 85.6 \\
\hline $4 \mathrm{H}-4,114-115$ & 27.14 & 81.6 \\
\hline $4 \mathrm{H}-4,136-137$ & 27.36 & 75.0 \\
\hline $4 \mathrm{H}-5,0-1$ & 27.50 & 75.3 \\
\hline $4 \mathrm{H}-5,16-17$ & 27.66 & 77.2 \\
\hline $4 \mathrm{H}-5,46-47$ & 27.96 & 79.6 \\
\hline $4 \mathrm{H}-5,63-64$ & 28.13 & 79.2 \\
\hline $4 \mathrm{H}-5,94-95$ & 28.44 & 86.0 \\
\hline $4 \mathrm{H}-5,114-115$ & 28.64 & 70.5 \\
\hline $4 \mathrm{H}-5,136-137$ & 28.86 & 88.6 \\
\hline $4 \mathrm{H}-6,0-1$ & 29.00 & 86.3 \\
\hline $4 \mathrm{H}-6,16-17$ & 29.16 & 70.0 \\
\hline $4 \mathrm{H}-6,46-47$ & 29.46 & 70.6 \\
\hline $4 \mathrm{H}-6,63-64$ & 29.63 & 78.8 \\
\hline $4 \mathrm{H}-6,94-95$ & 29.94 & 83.6 \\
\hline $4 \mathrm{H}-6,114-115$ & 30.14 & 82.7 \\
\hline $4 \mathrm{H}-6,136-137$ & 30.36 & 85.7 \\
\hline $4 \mathrm{H}-7,0-1$ & 30.50 & 88.0 \\
\hline $4 \mathrm{H}-7,16-17$ & 30.66 & 76.1 \\
\hline $4 \mathrm{H}-7,46-47$ & 30.96 & 74.4 \\
\hline $4 \mathrm{H}-7,63-64$ & 31.13 & 80.8 \\
\hline $5 \mathrm{H}-1,1-2$ & 31.01 & 79.6 \\
\hline $5 \mathrm{H}-1,20-21$ & 31.20 & 83.7 \\
\hline $5 \mathrm{H}-1,47-48$ & 31.47 & 88.8 \\
\hline SH-1, 94-95 & 31.94 & 93.5 \\
\hline $5 \mathrm{H}-1,114-115$ & 32.14 & 89.7 \\
\hline $5 \mathrm{H}-1,136-137$ & 32.36 & 88.0 \\
\hline $5 \mathrm{H}-2,1-2$ & 32.51 & 90.1 \\
\hline $5 \mathrm{H}-2,16-17$ & 32.66 & 93.0 \\
\hline $5 \mathrm{H}-2,47-48$ & 32.97 & 89.3 \\
\hline
\end{tabular}


Table 1 (continued).

\begin{tabular}{|c|c|c|c|c|c|}
\hline $\begin{array}{l}\text { Core, section, } \\
\text { interval }(\mathrm{cm})\end{array}$ & $\begin{array}{l}\text { Depth } \\
\text { (mbsf) }\end{array}$ & $\begin{array}{c}\mathrm{CaCO}_{3} \\
(\%)\end{array}$ & $\begin{array}{l}\text { Core, section, } \\
\text { interval }(\mathrm{cm})\end{array}$ & $\begin{array}{l}\text { Depth } \\
\text { (mbsf) }\end{array}$ & $\begin{array}{c}\mathrm{CaCO}_{3} \\
(\%)\end{array}$ \\
\hline $5 \mathrm{H}-2,63-64$ & 33.13 & 90.1 & $7 \mathrm{H}-1,16-17$ & 50.16 & 85.9 \\
\hline $5 \mathrm{H}-2,96-97$ & 33.46 & 95.4 & $7 \mathrm{H}-1,46-47$ & 50.46 & 85.6 \\
\hline $5 \mathrm{H}-2,114-115$ & 33.64 & 93.7 & $7 \mathrm{H}-1,63-64$ & 50.63 & 91.3 \\
\hline $5 \mathrm{H}-2,136-137$ & 33.86 & 88.6 & $7 \mathrm{H}-1,94-95$ & 50.94 & 92.0 \\
\hline $5 \mathrm{H}-3,1-2$ & 34.01 & 91.7 & $7 \mathrm{H}-1,114-115$ & 51.14 & 89.5 \\
\hline $5 \mathrm{H}-3,16-17$ & 34.16 & 83.6 & $7 \mathrm{H}-1,136-137$ & 51.36 & 89.5 \\
\hline $5 \mathrm{H}-3,47-48$ & 34.47 & 91.5 & $7 \mathrm{H}-2,0-1$ & 51.50 & 89.2 \\
\hline $5 \mathrm{H}-3,63-64$ & 34.63 & 90.4 & $7 \mathrm{H}-2,16-17$ & 51.66 & 83.2 \\
\hline $5 \mathrm{H}-3,94-95$ & 34.94 & 89.1 & $7 \mathrm{H}-2,46-47$ & 51.96 & 74.8 \\
\hline $5 \mathrm{H}-3,114-115$ & 35.14 & 89.8 & $7 \mathrm{H}-2,63-64$ & 52.13 & 73.2 \\
\hline $5 \mathrm{H}-3,136-137$ & 35.36 & 85.8 & $7 \mathrm{H}-2,94-95$ & 52.44 & 91.7 \\
\hline $5 \mathrm{H}-4,1-2$ & 35.51 & 88.7 & $7 \mathrm{H}-2,114-115$ & 52.64 & 91.5 \\
\hline $5 \mathrm{H}-4,16-17$ & 35.66 & 92.4 & $7 \mathrm{H}-2,136-137$ & 52.86 & 84.1 \\
\hline $5 \mathrm{H}-4,47-48$ & 35.97 & 88.2 & $7 \mathrm{H}-3,0-1$ & 53.00 & 75.9 \\
\hline $5 \mathrm{H}-4,63-64$ & 36.13 & 90.1 & $7 \mathrm{H}-3,16-17$ & 53.16 & 72.0 \\
\hline $5 \mathrm{H}-4,94-95$ & 36.44 & 84.9 & $7 \mathrm{H}-3,46-47$ & 53.46 & 66.1 \\
\hline $5 \mathrm{H}-4,114-115$ & 36.64 & 85.5 & $7 \mathrm{H}-3,60-61$ & 53.60 & 64.1 \\
\hline $5 \mathrm{H}-4,136-137$ & 36.86 & 83.6 & $7 \mathrm{H}-3,94-95$ & 53.94 & 67.1 \\
\hline $5 \mathrm{H}-5,1-2$ & 37.01 & 88.6 & $7 \mathrm{H}-3,114-115$ & 54.14 & 65.8 \\
\hline $5 \mathrm{H}-5,16-17$ & 37.16 & 83.0 & $7 \mathrm{H}-3,136-137$ & 54.36 & 67.6 \\
\hline $5 \mathrm{H}-5,47-48$ & 37.47 & 92.8 & $7 \mathrm{H}-4,0-1$ & 54.50 & 66.9 \\
\hline $5 \mathrm{H}-5,63-64$ & 37.63 & 83.5 & $7 \mathrm{H}-4,16-17$ & 54.66 & 65.5 \\
\hline SH-5, 94-95 & 37.94 & 78.2 & $7 \mathrm{H}-4,46-47$ & 54.96 & 78.0 \\
\hline $5 \mathrm{H}-5,114-115$ & 38.14 & 75.2 & $7 \mathrm{H}-4,60-61$ & 55.10 & 80.5 \\
\hline $5 \mathrm{H}-5,136-137$ & 38.06 & 78.0 & $7 \mathrm{H}-4,94-95$ & 55.44 & 83.2 \\
\hline $5 \mathrm{H}-6,1-2$ & 38.51 & 78.4 & $7 \mathrm{H}-4,114-115$ & 55.64 & 82.8 \\
\hline $5 \mathrm{H}-6,16-17$ & 38.66 & 70.8 & $7 \mathrm{H}-4,136-137$ & 55.86 & 83.3 \\
\hline $5 \mathrm{H}-6,47-48$ & 38.97 & 70.4 & $7 \mathrm{H}-5,0-1$ & 56.00 & 83.4 \\
\hline $5 H-6,63-64$ & 39.13 & 61.7 & $7 \mathrm{H}-5,16-17$ & 56.16 & 84.0 \\
\hline 5H-6, 94-95 & 39.44 & 73.3 & $7 \mathrm{H}-5,46-47$ & 56.46 & 81.7 \\
\hline 5H-6, 114-115 & 39.64 & 66.0 & $7 \mathrm{H}-5,60-61$ & 56.60 & 81.3 \\
\hline $5 \mathrm{H}-6,136-137$ & 39.86 & 70.7 & $7 \mathrm{H}-5,94-95$ & 56.94 & 77.4 \\
\hline $5 \mathrm{H}-7,1-2$ & 40.01 & 75.5 & $7 \mathrm{H}-5,114-115$ & 57.14 & 81.5 \\
\hline $5 \mathrm{H}-7,16-17$ & 40.16 & 80.8 & $7 \mathrm{H}-5,136-137$ & 57.36 & 86.6 \\
\hline $5 \mathrm{H}-7,47-48$ & 40.47 & 85.1 & $7 \mathrm{H}-6,0-1$ & 57.50 & 87.0 \\
\hline $5 \mathrm{H}-7,63-64$ & 40.63 & 85.5 & $7 \mathrm{H}-6,16-17$ & 57.66 & 85.4 \\
\hline $5 \mathrm{H}-7,91-92$ & 40.91 & 76.6 & $7 \mathrm{H}-6,46-47$ & 57.96 & 85.7 \\
\hline $6 \mathrm{H}-1,71-73$ & 41.21 & 88.1 & $7 \mathrm{H}-6,60-61$ & 58.10 & 85.1 \\
\hline $6 \mathrm{H}-1,93-95$ & 41.43 & 87.3 & $7 \mathrm{H}-6,94-95$ & 58.44 & 82.1 \\
\hline $6 \mathrm{H}-1,113-115$ & 41.63 & 87.3 & $7 \mathrm{H}-6,114-115$ & 58.64 & 79.8 \\
\hline $6 \mathrm{H}-1,136-138$ & 41.86 & 87.9 & $7 \mathrm{H}-6,136-137$ & 58.86 & 74.1 \\
\hline $6 \mathrm{H}-2,0-1$ & 42.00 & 86.2 & $7 \mathrm{H}-7,0-1$ & 59.00 & 54.4 \\
\hline $6 \mathrm{H}-2,16-17$ & 42.16 & 89.5 & $7 \mathrm{H}-7,16-17$ & 59.16 & 51.6 \\
\hline $6 \mathrm{H}-2,45-46$ & 42.45 & 85.6 & $7 \mathrm{H}-7,43-44$ & 59.43 & 78.9 \\
\hline $6 \mathrm{H}-2,72-74$ & 42.72 & 82.7 & $7 \mathrm{H}-7,60-61$ & 59.60 & 88.1 \\
\hline $6 \mathrm{H}-2,94-95$ & 42.94 & 85.1 & $8 \mathrm{H}-1,0-1$ & 59.50 & 90.5 \\
\hline $6 \mathrm{H}-2,114-115$ & 43.14 & 82.9 & $8 \mathrm{H}-1,16-17$ & 59.66 & 87.8 \\
\hline $6 \mathrm{H}-2,138-140$ & 43.38 & 88.0 & $8 \mathrm{H}-1,46-47$ & 59.96 & 93.0 \\
\hline $6 \mathrm{H}-3,0-1$ & 43.50 & 93.1 & $8 \mathrm{H}-1,63-64$ & 60.13 & 94.5 \\
\hline $6 \mathrm{H}-3,16-17$ & 43.66 & 94.4 & $8 \mathrm{H}-1,94-95$ & 60.44 & 90.8 \\
\hline $6 \mathrm{H}-3,45-46$ & 43.95 & 91.3 & $8 \mathrm{H}-1,114-115$ & 60.64 & 88.6 \\
\hline $6 \mathrm{H}-3,72-74$ & 44.22 & 92.7 & $8 \mathrm{H}-1,136-137$ & 60.86 & 92.5 \\
\hline $6 \mathrm{H}-3,94-95$ & 44.44 & 90.8 & $8 \mathrm{H}-2,0-1$ & 61.00 & 93.5 \\
\hline $6 \mathrm{H}-3,114-115$ & 44.64 & 83.5 & $8 \mathrm{H}-2,16-17$ & 61.16 & 94.7 \\
\hline $6 \mathrm{H}-3,138-140$ & 44.88 & 89.7 & $8 \mathrm{H}-2,46-47$ & 61.46 & 94.2 \\
\hline $6 \mathrm{H}-4,0-1$ & 45.00 & 90.1 & $8 \mathrm{H}-2,63-64$ & 61.63 & 82.1 \\
\hline $6 \mathrm{H}-4,16-17$ & 45.16 & 89.5 & $8 \mathrm{H}-2,94-95$ & 61.94 & 88.0 \\
\hline $6 \mathrm{H}-4,45-46$ & 45.45 & 91.7 & $8 \mathrm{H}-2,114-115$ & 62.14 & 83.8 \\
\hline $6 \mathrm{H}-4,72-74$ & 45.72 & 90.5 & $8 \mathrm{H}-2,136-137$ & 62.36 & 83.9 \\
\hline $6 \mathrm{H}-4,94-95$ & 45.94 & 92.6 & $8 \mathrm{H}-3,0-1$ & 62.50 & 80.3 \\
\hline $6 \mathrm{H}-4,114-115$ & 46.14 & 90.7 & $8 \mathrm{H}-3,16-17$ & 62.66 & 78.8 \\
\hline $6 \mathrm{H}-4,138-140$ & 46.38 & 95.0 & $8 \mathrm{H}-3,46-47$ & 62.96 & 84.5 \\
\hline $6 \mathrm{H}-5,0-1$ & 46.50 & 88.5 & $8 \mathrm{H}-3,63-64$ & 63.13 & 82.5 \\
\hline $6 \mathrm{H}-5,16-17$ & 46.66 & 91.3 & $8 \mathrm{H}-3,94-95$ & 63.44 & 69.7 \\
\hline $6 \mathrm{H}-5,45-46$ & 46.95 & 92.6 & $8 \mathrm{H}-3,114-115$ & 63.64 & 55.8 \\
\hline $6 \mathrm{H}-5,72-74$ & 47.22 & 92.8 & $8 \mathrm{H}-3,136-137$ & 63.86 & 77.6 \\
\hline $6 \mathrm{H}-5,94-95$ & 47.44 & 94.9 & $8 \mathrm{H}-4,0-1$ & 64.00 & 77.9 \\
\hline $6 \mathrm{H}-5,114-115$ & 47.64 & 95.5 & $8 \mathrm{H}-4,16-17$ & 64.16 & 82.1 \\
\hline $6 \mathrm{H}-5,138-140$ & 47.88 & 92.7 & $8 \mathrm{H}-4,46-47$ & 64.46 & 75.8 \\
\hline $6 \mathrm{H}-6,0-1$ & 48.00 & 92.9 & $8 \mathrm{H}-4,63-64$ & 64.63 & 69.2 \\
\hline $6 \mathrm{H}-6,16-17$ & 48.16 & 87.6 & $8 \mathrm{H}-4,94-95$ & 64.94 & 40.2 \\
\hline $6 \mathrm{H}-6,45-46$ & 48.45 & 92.6 & $8 \mathrm{H}-4,114-115$ & 65.14 & 52.3 \\
\hline $6 \mathrm{H}-6,72-74$ & 48.72 & 91.6 & $8 \mathrm{H}-4,136-137$ & 65.36 & 72.0 \\
\hline $6 \mathrm{H}-6,94-95$ & 48.94 & 90.9 & $8 \mathrm{H}-5,0-1$ & 65.50 & 78.0 \\
\hline $6 \mathrm{H}-6,114-115$ & 49.14 & 90.7 & $8 \mathrm{H}-5,16-17$ & 65.66 & 77.5 \\
\hline $6 \mathrm{H}-6,138-140$ & 49.38 & 92.5 & $8 \mathrm{H}-5,46-47$ & 65.96 & 78.0 \\
\hline $7 \mathrm{H}-1,0-1$ & 50.00 & 90.0 & $8 \mathrm{H}-5,63-64$ & 66.13 & 79.4 \\
\hline
\end{tabular}

\begin{tabular}{|c|c|c|}
\hline $\begin{array}{l}\text { Core, section, } \\
\text { interval }(\mathrm{cm})\end{array}$ & $\begin{array}{l}\text { Depth } \\
\text { (mbsf) }\end{array}$ & $\begin{array}{c}\mathrm{CaCO}_{3} \\
(\%)\end{array}$ \\
\hline $8 \mathrm{H}-5,94-95$ & 66.44 & 83.3 \\
\hline $8 \mathrm{H}-5,114-115$ & 66.64 & 85.7 \\
\hline $8 \mathrm{H}-5,136-137$ & 66.86 & 82.6 \\
\hline $8 \mathrm{H}-6,0-1$ & 67.00 & 79.6 \\
\hline $8 \mathrm{H}-6,16-17$ & 67.16 & 72.6 \\
\hline $8 \mathrm{H}-6,46-47$ & 67.46 & 77.0 \\
\hline $8 \mathrm{H}-6,63-64$ & 67.63 & 79.4 \\
\hline $8 \mathrm{H}-6,94-95$ & 67.94 & 78.6 \\
\hline $8 \mathrm{H}-6,114-115$ & 68.14 & 83.0 \\
\hline $8 \mathrm{H}-6,136-137$ & 68.36 & 86.1 \\
\hline $8 \mathrm{H}-7,0-1$ & 68.50 & 86.6 \\
\hline $8 \mathrm{H}-7,16-17$ & 68.66 & 85.9 \\
\hline $8 \mathrm{H}-7,46-47$ & 68.96 & 79.5 \\
\hline $8 \mathrm{H}-\mathrm{CC}, 0-1$ & 69.03 & 81.8 \\
\hline $9 \mathrm{H}-1,0-1$ & 69.00 & 83.8 \\
\hline $9 \mathrm{H}-1,16-17$ & 69.16 & 88.0 \\
\hline $9 \mathrm{H}-1,46-47$ & 69.46 & 88.7 \\
\hline $9 \mathrm{H}-1,64-65$ & 69.64 & 89.0 \\
\hline $9 \mathrm{H}-1,94-95$ & 69.94 & 87.4 \\
\hline $9 \mathrm{H}-1,114-115$ & 70.14 & 84.2 \\
\hline $9 \mathrm{H}-1,136-137$ & 70.36 & 88.8 \\
\hline $9 \mathrm{H}-2,0-1$ & 70.50 & 89.0 \\
\hline $9 \mathrm{H}-2,16-17$ & 70.66 & 89.3 \\
\hline $9 \mathrm{H}-2,46-47$ & 70.96 & 88.5 \\
\hline $9 \mathrm{H}-2,64-65$ & 71.14 & 80.9 \\
\hline $9 \mathrm{H}-2,94-95$ & 71.44 & 88.7 \\
\hline $9 \mathrm{H}-2,114-115$ & 71.64 & 85.0 \\
\hline $9 \mathrm{H}-2,136-137$ & 71.86 & 92.2 \\
\hline $9 \mathrm{H}-3,0-1$ & 72.00 & 92.5 \\
\hline $9 \mathrm{H}-3,16-17$ & 72.16 & 88.8 \\
\hline $9 \mathrm{H}-3,47-48$ & 72.47 & 94.0 \\
\hline $9 \mathrm{H}-3,64-65$ & 72.64 & 93.9 \\
\hline $9 \mathrm{H}-3,94-95$ & 72.94 & 90.8 \\
\hline $9 \mathrm{H}-3,114-115$ & 73.14 & 93.0 \\
\hline $9 \mathrm{H}-3,136-137$ & 73.36 & 93.2 \\
\hline $9 \mathrm{H}-4,0-1$ & 73.50 & 89.1 \\
\hline $9 \mathrm{H}-4,16-17$ & 73.66 & 92.6 \\
\hline $9 \mathrm{H}-4,46-47$ & 73.96 & 93.3 \\
\hline $9 \mathrm{H}-4,64-65$ & 74.14 & 92.5 \\
\hline $9 \mathrm{H}-4,94-95$ & 74.44 & 91.3 \\
\hline $9 \mathrm{H}-4,114-115$ & 74.64 & 91.9 \\
\hline $9 \mathrm{H}-4,136-137$ & 74.86 & 89.6 \\
\hline $9 \mathrm{H}-5,0-1$ & 75.00 & 90.8 \\
\hline $9 \mathrm{H}-5,16-17$ & 75.16 & 87.6 \\
\hline $9 \mathrm{H}-5,46-47$ & 75.46 & 92.2 \\
\hline $9 \mathrm{H}-5,64-65$ & 75.64 & 87.6 \\
\hline $9 \mathrm{H}-5,94-95$ & 75.94 & 90.7 \\
\hline $9 \mathrm{H}-5,114-115$ & 76.14 & 93.0 \\
\hline $9 \mathrm{H}-5,136-137$ & 76.36 & 92.8 \\
\hline $9 \mathrm{H}-6,0-1$ & 76.50 & 92.1 \\
\hline $9 \mathrm{H}-6,16-17$ & 76.66 & 91.5 \\
\hline $9 \mathrm{H}-6,46-47$ & 76.96 & 92.0 \\
\hline $9 \mathrm{H}-6,64-65$ & 77.14 & 92.9 \\
\hline $9 \mathrm{H}-6,94-95$ & 77.44 & 93.2 \\
\hline $9 \mathrm{H}-6,114-115$ & 77.64 & 92.4 \\
\hline $9 \mathrm{H}-6,136-137$ & 77.86 & 89.6 \\
\hline $9 \mathrm{H}-\mathrm{CC}, 0-1$ & 78.00 & 91.2 \\
\hline $9 \mathrm{H}-\mathrm{CC}, 16-17$ & 78.16 & 92.3 \\
\hline
\end{tabular}




\section{Volume magnetic susceptibility $\left(10^{-6} \mathrm{cgs}\right)$}
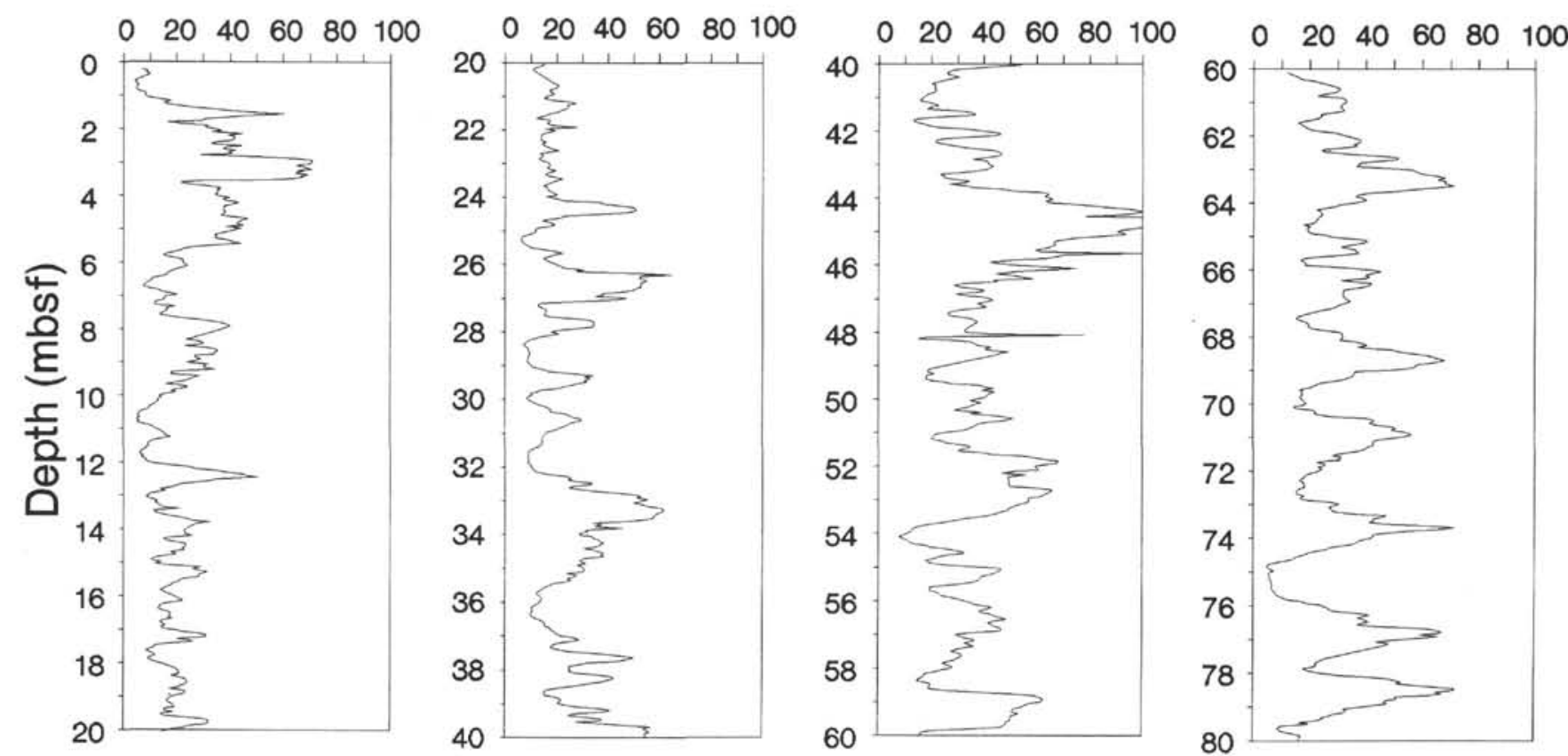

Volume magnetic susceptibility $\quad\left(10^{-6} \mathrm{cgs}\right)$
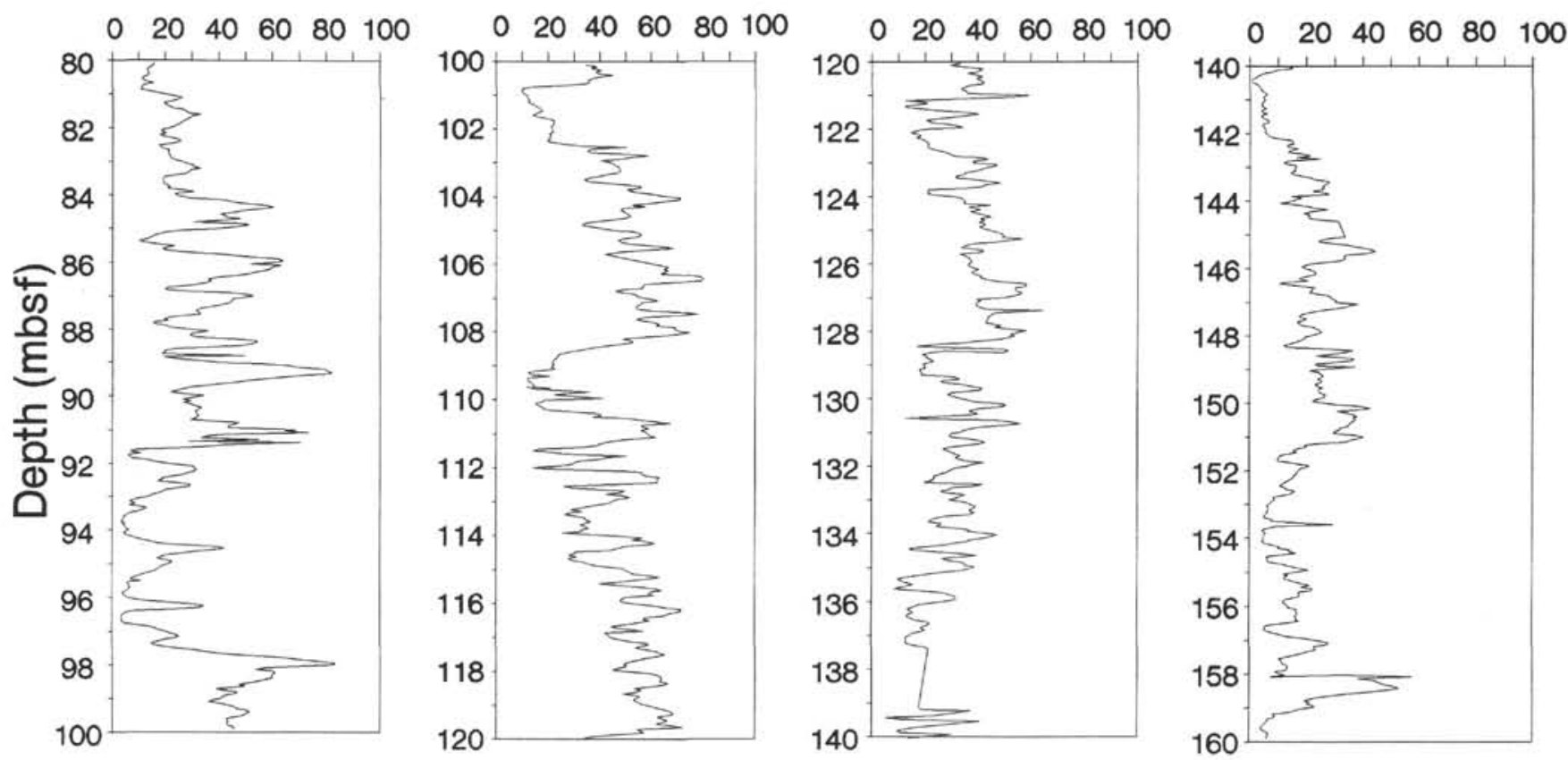

Figure 3. Volume magnetic susceptibility log of Hole 745B, divided into 20 -m intervals. 
Volume magnetic susceptibility $\left(10^{-6} \mathrm{cgs}\right)$

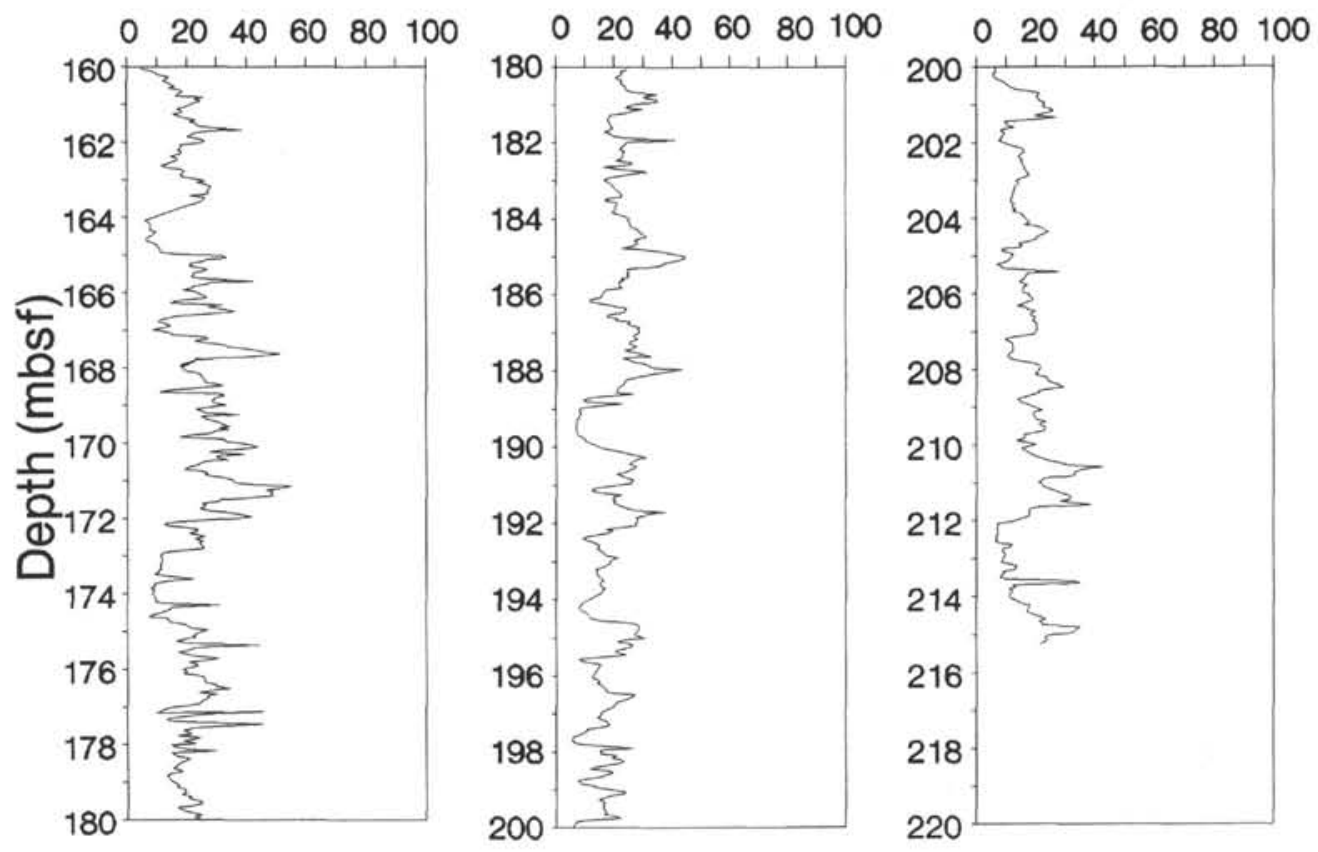

Figure 3 (continued). 